\title{
Theory of neutral divacancy in SiC: a defect for spintronics
}

\author{
Adam Gali, Andreas Gällström, Nguyen Tien Son and Erik Janzén
}

\section{Linköping University Post Print}

N.B.: When citing this work, cite the original article.

Original Publication:

Adam Gali, Andreas Gällström, Nguyen Tien Son and Erik Janzén, Theory of neutral divacancy in SiC: a defect for spintronics, 2010, Materials Science Forum, Vols. 645-648, 395-397.

http://dx.doi.org/10.4028/www.scientific.net/MSF.645-648.395

Copyright: Trans Tech Publications

Postprint available at: Linköping University Electronic Press

http://urn.kb.se/resolve?urn=urn:nbn:se:liu:diva-62704 


\title{
Theory of neutral divacancy in SiC: a defect for spintronics
}

\author{
Adam Gali $^{1)}$, A. Gallström ${ }^{2)}$, N.T. Son ${ }^{2)}$, E. Janzén ${ }^{2)}$ \\ 1) Department of Atomic Physics, Budapest University of Technology and Economics, \\ Budapest, Budafoki út 8., $\mathrm{H}-1111$, Hungary \\ 2) Department of Physics, Chemistry and Biology, Linköping University, Linköping, S- \\ 58183 , Sweden \\ agali@eik.bme.hu
}

We have recently identified the neutral divacancy in $4 \mathrm{H}-\mathrm{SiC}$ based on the agreement between the calculated and measured hyperfine signals [1]. This defect has the unique property that the ground state is a high spin $S=1$ state with a zero field splitting of $D \approx 1.3 \mathrm{GHz}$ [2]. The so-called 'divacancy' [3] and UD-2 photoluminescence (PL) [4] signals were associated with this defect, with zero-phonon lines (ZPL) at $\sim 1.0$ and $\sim 1.1 \mathrm{eV}$ in $4 \mathrm{H}-\mathrm{SiC}$. The 'divacancy' transition can be also detected in infrared (IR) absorption [3]. It is also known that $\sim 1.1 \mathrm{eV}$ excitation can enhance the electron paramagnetic signal (EPR) of the divacancy [1,5]. Detailed knowledge about the ground and excited states of this defect is crucial to understand the basic processes associated with this defect. We studied the ground and excited states by advanced quantum mechanical calculations and group theory analysis. We will show that the unique properties of this defect can be promising for optical manipulation of the electron and nuclear spin states of the defect.

We investigated the ground and excited states by advanced ab initio density functional calculations (DFT) and beyond. The fine electron structure and levels were studied by group theory. We obtained the spin density distribution of the axial divacancy with $\mathrm{C}_{3 \mathrm{v}}$ symmetry in $4 \mathrm{H}-\mathrm{SiC}$ by supercell plane wave calculations using DFT within local density approximation. The absorption spectrum of the neutral divacancy was calculated in a cubic nano-SiC with a diameter of $1.4 \mathrm{~nm}$ by time-dependent DFT with applying a hybrid functional in the kernel. We were able to calculate the characteristic vertical absorption energies accurately and also to estimate the zero-phonon lines of the defect.

The calculated spin density distribution for the cubic axial configuration is shown in Fig. 1a,b. Apparently, the spin density decays very fast and is practically negligible outside the radius of $5 \AA$ from the core of the defect. The spin density is mostly detectable on ${ }^{13} \mathrm{C}$ isotopes via hyperfine interaction between the electron and nuclear spins and the values are typically $<10 \sim \mathrm{MHz}$ except the first neighbor $\mathrm{C}$-atoms near the carbon vacancy. As was demonstrated earlier for nitrogen-vacancy defect in diamond, spin-echo technique can be used to entangle the electron and nuclear spins of the system [6]. The fast decay of the spin density is important criteria in order to avoid the fast decoherence of the entangled states which is fulfilled for the divacancy in SiC. The nuclear spin bath of ${ }^{29} \mathrm{Si}$ isotopes can represent a problem but it is possible to grow ${ }^{28} \mathrm{Si}$-isotope riched $\mathrm{SiC}$ samples. In spintronics applications, the electron and nuclear spins are manipulated by optical excitations, so next we focus on the fine electron structure of the defect.

Application of group theory can be very useful to understand the electronic structure of defects. We consider the $\mathrm{C}_{3 \mathrm{v}}$ symmetry of the divacancy which occurs in $3 \mathrm{C}-\mathrm{SiC}$ and for the axial configurations in hexagonal $\mathrm{SiC}$. The three $\mathrm{C}$ and $\mathrm{Si}$ dangling bonds create six single particle states with $a_{1(1)}(2) a_{1(2)}(2) e_{(1)}(2) e_{(2)}(0)$ occupations in the ground state. According to the supercell calculations the $a_{1(1)}$ state falls deeply in VB and can be ignored, whereas $a_{1(2)}$ is resonant with the valence band edge $(\mathrm{VBM})$. Both $e_{(1)}$ and $e_{(2)}$ states fall in the fundamental gap; $e_{(1) /(2)}$ is predominantly localized on $\mathrm{C} / \mathrm{Si}$ dangling bonds. The possible multiplets of the $a_{1(2)}(2) e_{(1)}(2) e(0)$ configuration is the triplet ${ }^{3} \mathrm{~A}_{2}$ and the ${ }^{1} \mathrm{E},{ }^{1} \mathrm{~A}_{1}$ singlets (Fig. 1c,d). The ground state is the high-spin ${ }^{3} \mathrm{~A}_{2}$, giving rise large spin density on the nearest C-atoms (see Fig. 1a,b). In our group theory analysis we could prove that the order of levels is ${ }^{3} \mathrm{~A}_{2},{ }^{1} \mathrm{E},{ }^{1} \mathrm{~A}_{1}$. The excitation can occur from the resonant $a_{1(2)}$ state resulting in $a_{1(2)}(1) e_{(1)}(3) e(0)$. This will result in ${ }^{3} \mathrm{E}$ and ${ }^{1} \mathrm{E}$ multiplets where ${ }^{1} \mathrm{E}$ is above ${ }^{3} \mathrm{E}$ (Fig. 1d). The spin-conserving excitation 
occurs between ${ }^{3} \mathrm{~A}_{2} \rightarrow{ }^{3} \mathrm{E}$. The calculated vertical absorption energy is $1.18 \mathrm{eV}$ for this process. Due to excitation the atoms relaxed to a slightly distorted $\mathrm{C}_{3 \mathrm{v}}$ symmetry. The calculated ZPL energy is $\sim 1.05$ $\mathrm{eV}$ that is close to the 'divacancy' ZPL data. The slight distortion indicates minutely splitted ZPL lines with somewhat different polarization dependence as indeed measured. The fine structure of the ${ }^{3} \mathrm{~A}_{2}$ state has spin-spin splitting while ${ }^{3} \mathrm{E}$ state has additionally spin-orbit splitting at low temperature (Fig 1e.). Presumably, the ${ }^{1} \mathrm{~A}_{1}$ state is close to the ${ }^{3} \mathrm{E}$ state. The appropriate $M_{S}= \pm 1$ states of ${ }^{3} \mathrm{E}$ can couple with ${ }^{1} \mathrm{~A}_{1}$ state giving rise non-radiative recombination which also couples to the $M_{S}=0$ of ${ }^{3} \mathrm{~A}_{2}$ ground state (Fig. 1d,e). Finally, optical excitation will change the $M_{S}= \pm 1$ of ${ }^{3} \mathrm{~A}_{2}$ to $M_{S}=0$. This will enhance the EPR signal under light illumination at $\sim 1.1 \mathrm{eV}$ because the population of $M_{S}=0$ state is increased with giving rise higher probability of $M_{S}=0 \rightarrow \pm 1$ EPR transitions. Thus, the electron spin state of the defect can be manipulated optically.

Beside this process other excitation channels are also feasible. The resonant $a_{1(2)}$ state may be occupied first by an electron from VBM after optical excitation resulting in a [divacancy(-)+hole] excited configuration. Then, the excited electron will recombine with the bound hole and not with the $a_{1(2)}$ state. This may explain that two PL centers can be associated with the neutral divacancy with very similar energies. We found other intriguing optical properties of the divacancy. It is possible to excite not just to $a_{1(2)}(1) e_{(1)}(3) e(0)$ but to $a_{1(2)}(1) e_{(1)}(2) e(1)$ and $a_{1(2)}(2) e_{(1)}(1) e(1)$ configurations. The calculated vertical absorption energies of these processes are 1.85 and $2.34 \mathrm{eV}$, respectively. These excitations cause a significant distortion from $\mathrm{C}_{3 \mathrm{v}}$ symmetry and large relaxation energy $(\sim 0.46 \mathrm{eV})$. The estimated ZPL energies are $\sim 1.39$ and $\sim 1.88 \mathrm{eV}$, respectively. The former is close to UD-4 ZPL's energies $(\sim 1.43 \mathrm{eV})$ [7] but no connection between UD-4 and UD-2 or the 'divacancy' PL signals have been shown. Our study revealed that this defect can be excited both far IR and visible light.

References

[1] N.T. Son et al., Phys. Rev. Lett., 96, 055501 (2006).

[2] V.S. Vainer and V.A. Il'in, Sov. Phys. Sol. State, 23, 2126 (1981).

[3] B. Magnusson and E. Janzén, Mat. Sci. Forum, 483-485, 341 (2005).

[4] W.E. Carlos et al., Physica B, 340-342, 151 (2003).

[5] Th. Lingner et al., Phys. Rev. B, 64, 245212 (2001).

[6] L. Childress et al., Science, 314, 281 (2006).

[7] A. Gallström et al., Mat. Sci. Forum a) top

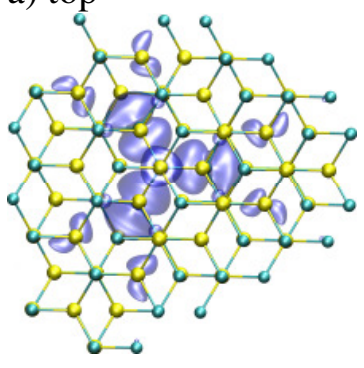

b) side

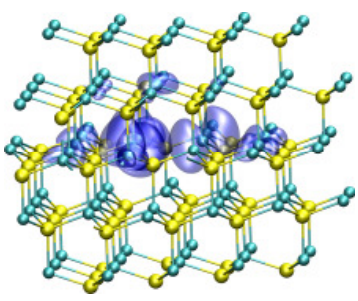

c)

d)

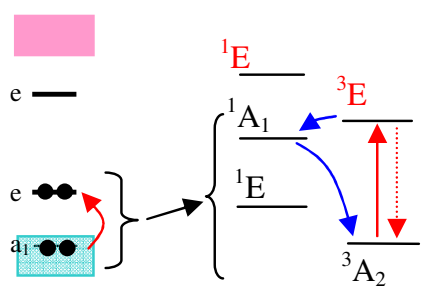

e)

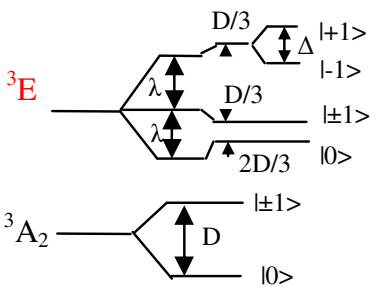

Fig. 1. a) top b) side views of the calculated spin density isosurface of the ground state of divacancy at axial cubic site in 4H-SiC. Cyan(yellow) balls represent carbon(silicon) atoms. c) Schematic sketch of the single particle states in the gap and its occupation in the ground state. Cyan/rose rectangles are valence/conduction band states. Red arrow shows the excitation. d) Schematic sketch of the order of multiplets. Red: excited configuration. Red/blue arrows represent radiative/non-radiative transitions. e) Fine structure of levels due to spin-spin (D, $\Delta$ ) and spin-orbit $(\lambda)$ interaction. 